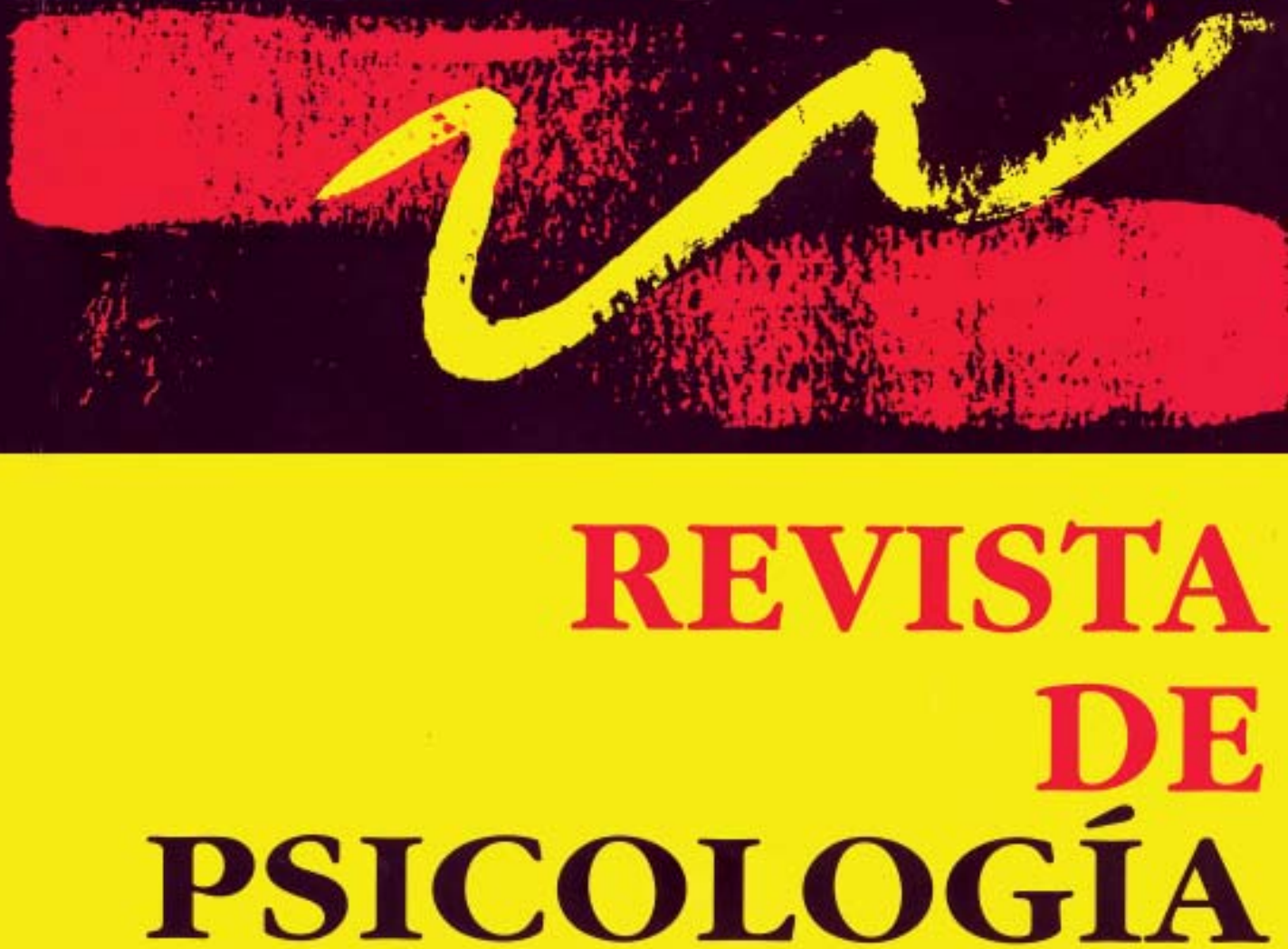

Silencio como secuela de la guerra interna en Perú

Comportamiento antisocial en menores escolares

e indigentes: influencia del vecindario y de los padres

La unidimensionalidad de un instrumento de medición: perspectiva factorial

Calidad de la amistad en niños de 8 a 10 años de una institución pública

Afrontamiento a la enfermedad crónica:

estudio en pacientes con insuficiencia renal crónica terminal
Javier Iguíñiz Echeverría

Ana Lilia Banda Castro y Martha Frías Armenta

Andrés Burga León

Doris Argumedo Bustinza y Carla Albornoz Álvarez

Mónica Cassaretto y Rosario Paredes

Reseña 


\section{REVISTA DE PSICOLOGÍA}

\section{Vol. XXIV. Primer semestre 2006 N $^{\circ} 1$ \\ CONTENIDO}

\section{ARTÍCULOS}

Javier Iguíniz Echeverría. Silencio como secuela de la guerra interna en Perú.

Ana Lilia Banda Castro y Martha Frías Armenta. Comportamiento antisocial en menores escolares e indigentes: influencia del vecindario y de los padres.

Andrés Burga León. La unidimensionalidad de un instrumento de medición: perspectiva factorial.

Doris Argumedo Bustinza y Carla Albornoz Álvarez. Calidad de la amistad en niños de 8 a 10 años de una institución pública.

Mónica Cassaretto y Rosario Paredes. Afrontamiento a la enfermedad crónica: estudio en pacientes con insuficiencia renal crónica terminal.

RESEÑA 
Revista de Psicología de la PUCP. Vol. XXIV, 1, 2006

\title{
Comportamiento antisocial en menores escolares e indigentes: influencia del vecindario y de los padres
}

\author{
Ana Lilia Banda Castro ${ }^{1}$ y Martha Frías Armenta ${ }^{2}$ \\ Universidad de Sonora
}

\begin{abstract}
Se analizó la influencia del vecindario y la familia en el comportamiento antisocial de menores. Los participantes fueron 192 menores, 96 con antecedentes de indigencia y 96 escolares no indigentes. Se administraron la Escala de Comportamiento Antisocial (Castell, Frías, Corral \& Sotomayor, 2000) y las Escalas de Comportamiento Adictivo (Reich \& Herjanic, 1989; Vazsonyi, Pickering, Junger \& Hessing, 2001). Se calcularon frecuencias de medias, desviaciones estándar y modelamiento estructural. Los resultados mostraron que la conducta antisocial y adictiva estaba influenciada por el comportamiento antisocial del padre, el comportamiento adictivo de la madre y el vecindario. Para los menores indigentes, el comportamiento antisocial estaba directamente influido por su comportamiento adictivo y el comportamiento antisocial del padre. Para el grupo de estudiantes el comportamiento antisocial estaba influido por el vecindario, por su comportamiento adictivo y por el comportamiento antisocial del padre. Para ambos grupos el vecindario y el comportamiento adictivo de la madre influyeron directamente en el comportamiento adictivo de los mismos. Palabras clave: comportamiento antisocial, niños indigentes.
\end{abstract}

\section{Antisocial behavior in students and homeless children: Influence of neighborhood and parents}

The aim of this work was to analyze the influence of neighbors and parents on children's antisocial behavior. The participants were 96 homeless children and 96 students. The instruments applied were the Scale of Antisocial Behavior (Castell, Frías, Corral \& Sotomayor, 2000) and the Scales of Addictive Behavior (Reich \& Herjanic, 1989; Vazsonyi, Pickering, Junger \& Hessing, 2001). First univariate statistics were obtained, after a model was tested using structural equations modeling. The data showed that children's antisocial and addictive behavior was affected by father's antisocial behavior, mother's addictive behavior and neighborhood. For homeless children their antisocial behavior was influenced directly by their addictive behavior and father's antisocial behavior. For students, neighborhood, father's antisocial behavior and children's addictive behavior influenced on children's antisocial behavior. In both groups the neighborhood and mother's addictive behavior influenced directly children's addictive behavior.

Keywords: antisocial behavior, homeless children.

Profesora de asignatura. Doctora en Ciencias Sociales por la Universidad Autónoma de Sinaloa. Ha realizado investigaciones sobre comportamiento indigente, valores y organizaciones. Correo electrónico: albanda@psicom.uson.mx

2 Profesora titular. Doctora en Psicología por la Universidad de Arizona. Ha realizado investigación sobre violencia familiar, delincuencia juvenil y educación. Correo electrónico: marthafrias@ sociales.uson.mx 

El comportamiento antisocial, de acuerdo con Mcevoy y Welker (2000), se refiere a todos aquellos comportamientos indeseables de los menores que no necesariamente son violentos y tampoco se relacionan con el consumo de drogas, pero que se vinculan directamente con un pobre rendimiento escolar, deficiencias de atención y aprendizaje, por mencionar sólo algunos.

Galambos, Barker y Almeida (2003) han diferenciado dos perspectivas del comportamiento antisocial: a) cuando el individuo que lo manifiesta lo externaliza y b) cuando lo dirige hacia sí mismo, es decir, lo internaliza. En el caso de que un menor externalice su comportamiento manifiesta señales de conductas como son fumar, cometer pequeños hurtos, ingerir drogas, desobedecer, cometer acciones que atentan contra terceros, etcétera. Si el menor internaliza su comportamiento se presentan síntomas como depresión, ansiedad, introversión o temor.

El comportamiento antisocial representa manifestaciones que comprenden acciones aversivas para terceros e incompatibles con las normas y costumbres de la sociedad o que atentan contra la integridad de terceros o contra la propiedad privada como: tirar basura, consumir y comercializar drogas, cometer robos, ocasionar lesiones a terceros, convertirse en homicida, manifestar crueldad hacia los individuos o los animales, provocar incendios, utilizar las mentiras de forma sistemática, involucrarse en peleas o ataques físicos, escapar de la escuela o del hogar, por mencionar algunos ejemplos (Ángel, Gaviria \& Restrepo, 2003; Cuevas, 2003; Ruiz, 1998). 
El Instituto Nacional de Estadística, Geografía e Informática en México (INEGI) (2002) indica que durante 2001 han sido arrestados 163,995 menores en todo el país y 7130 en el estado de Sonora, de los cuales 2,694 fueron puestos a disposición del Consejo Tutelar para Menores (COTUME) (INEGI, 2002). Al margen de estas cifras quedan los actos antisociales que nunca son reportados por miedo o por considerar que no tiene objeto hacerlo, lo cual hace pensar que los actos antisociales podrían alcanzar cifras sorprendentes que desafortunadamente van en incremento.

El comportamiento adictivo se caracteriza por el consumo reiterado y continuo de sustancias con el fin de experimentar los efectos que produce o bien para evitar los síntomas desagradables que se presentan si no se consumen (Velasco, 2003). El National Institute on Drug Abuse (NIDA) (2006) define el comportamiento adictivo como el empleo de drogas de forma regular y repetida, ya sea tabaco, alcohol, cocaína, inhalantes o heroína, por mencionar algunas.

Los efectos de condiciones de riesgo (la violencia comunitaria, la violencia e inseguridad transmitida por condiciones de guerra, la marginalidad social, el abandono, la desintegración familiar o bien la interacción del menor con padres o cuidadores adictos o alterados mentalmente) han sido referidos por Garbarino (2001) específicamente al identificar cuándo y cómo los niños se ven expuestos a las más extralimitadas experiencias de violencia comunitaria como para rebasar los límites de su potencialidad para sobreponerse a la misma.

Ya que, de acuerdo con Garbarino (2001), estados crónicos de vivencias de riesgo y de violencia transforman el comportamiento de los soldados de diversos lugares y tiempos en comportamiento psicopático y agresivo. Esto es digno de ser analizado, ya que si la reacción de estos adultos es tan desfavorable, cuando los mismos han tenido oportunidad de involucrarse con situaciones 
muy favorables durante la infancia y la adolescencia, se considera que los efectos de la adversidad crónica, el estrés, la falta de apego emocional y de educación en aspectos morales, desde edades tempranas puede magnificar la respuesta de estos menores que se encuentran en desarrollo.

Para Garbarino (2001) los factores ambientales como la pobreza, el racismo, la ausencia de padres, la configuración de los mapas sociales con carencias afectivas y carentes de esquemas morales efectivos, así como los trastornos psicológicos padecidos crónicamente por los menores en forma de miedo, inseguridad e impotencia, constituyen aspectos que día a día hacen que la humanidad se enfrente a individuos susceptibles de cometer los homicidios colectivos o individuales más terribles e incluso de atentar contra ellos mismos.

Otro aspecto teórico que apoya los datos de esta investigación lo aportan Tolan, Gorman-Smith y Henry (2003), los cuales proponen un modelo multinivel sustentado por la teoría ecológica del desarrollo de Bronfenbrenner (1987). Este modelo está integrado por factores socio-ecológicos como son la estructura comunitaria y los procesos sociales del vecindario, la interacción paterna, la influencia de pares y el comportamiento violento que tienden a afectar la manifestación de violencia hacia el entorno por parte de los jóvenes en forma de asalto, asalto con agravantes, intento de asesinato o lesiones, por citar algunos ejemplos.

Tanto los adultos como los menores suelen cometer actos antisociales frecuentemente. De acuerdo con Beyers, Loeber, Wikström y Stouthamer-Loeber (2001) y Wikström y Loeber (2000) existe una relación directa entre la pobreza que caracteriza a los vecindarios y la manifestación de comportamiento antisocial. Se ha visto que cuanto más desfavorable es el vecindario, más alto es el índice de comportamiento antisocial que tienden a mostrar 
sus integrantes; al parecer dentro de las sociedades se va haciendo común desenvolverse dentro de una dinámica de antisocialidad. En este sentido, Ceballo, Dahl, Aretakis y Ramírez (2001) han señalado que la violencia dentro de las comunidades tiende a pasar desapercibida, a olvidarse o minimizarse, ya que la conducta violenta se vuelve una pauta de comportamiento usual dentro de los integrantes de las comunidades.

Además de la importancia que tiene el entorno para propiciar la antisocialidad existen datos como los aportados por Barrera et al. (2002), Galambos et al. (2003) y Rosario, Salzinger, Feldman y Nq-Mak (2003) que señalan que los compañeros de los vecindarios ejercen una notable influencia para que incluso menores que no habían mostrado comportamiento antisocial tiendan a la externalización del comportamiento (impulsividad, consumo de alcohol o drogas y comportamiento antisocial). De acuerdo con Tolan, Gorman-Smith \& Henry (2003) los vecindarios afectan indirectamente el funcionamiento familiar, en virtud de que el ingreso del menor a una pandilla o grupo de pares reduce la interacción del mismo con la familia.

Diversas investigaciones (Frías, 2004; Frías, Ramírez, Soto, Castell \& Corral, 2000; Pinderhughes, Nix, Foster \& Jones, 2001) plantean que las situaciones que más afectan la conducta antisocial de los menores se relacionan con la violencia entre los padres (Fergusson \& Horwood, 1998; Schwartz, Dodge, Pettit \& Bates, 2000) y con el abuso físico hacia los hijos (Frías, Corral, Moreno \& Rodríguez, 2000; Frías, Figueredo, Corral, Peña \& Quiroz, 2002; Frías, Ramírez et al., 2000; Frías, Sotomayor, Varela, Zaragoza, Banda \& García, 2000), aunado esto con las características de incivilidad de los vecindarios y los ambientes familiares que son responsables de las actitudes negativas de los adolescentes y de la inhibición de habilidades sociales. 
Se ha podido constatar que los menores con comportamiento adictivo (al alcohol o a las drogas) buscan la interacción con individuos con idéntico comportamiento, ya que estos últimos son fuente de aceptación del comportamiento adictivo o permiten el abastecimiento de las sustancias adictivas (Early Child Care Research Network Rockville Maryland, 2003; Garnier \& Stein, 2002; Hussong \& Hicks, 2003; Natera, Mora \& Tiburcio, 1999; Schwartz et al., 2000). Asimismo, el comportamiento antisocial suele ser propiciado por la interacción con amigos o compañeros que dan muestra del mismo (Barrera et al., 2002; Galambos et al., 2003; Rosario et al., 2003).

Generalmente el comportamiento adictivo se encuentra como mediador para que los individuos cometan toda clase de comportamientos antisociales, tal es el caso de las investigaciones practicadas por Frías (2002), Frías, Corral et al. (2000) y Natera et al. (1999) que refieren que además del problema adictivo los menores presentan otros tipos de problemas como comportamiento antisocial o aislamiento familiar.

Existen evidencias contundentes de que los padres influyen notablemente sobre el comportamiento antisocial o adictivo de los hijos, especialmente cuando dichos padres muestran tendencia al comportamiento antisocial (Frías, 2002; NICHD, 2003), al comportamiento adictivo (de alcohol o drogas) (Frías, 2002; NICHD, 2003; Nyamathi, Longshore, Keenan, Lesser \& Leake, 2001; Stein, Burden Leslie \& Nyamathi, 2002) y cuando participan en procesos de violencia intrafamiliar (Tyler \& Cause, 2002).

La presente investigación se planteó las hipótesis que identificaron: a) la influencia que ejerce el comportamiento antisocial del padre y el vecindario en el comportamiento antisocial del menor, b) la influencia del comportamiento adictivo de la madre y del vecindario en el comportamiento adictivo del menor, y c) la 
relación existente entre el comportamiento adictivo del menor y su comportamiento antisocial.

En esta investigación se buscó identificar si las manifestaciones de comportamiento antisocial y de comportamiento adictivo de dos grupos de menores se relacionan con la interacción familiar caracterizada por el comportamiento adictivo de la madre y el comportamiento antisocial del padre, con el vecindario en donde prevalece el comportamiento adictivo y antisocial, así como con la violencia comunitaria.

\section{Metodología}

\section{Participantes}

La muestra estuvo integrada por dos grupos de menores de la Ciudad de Hermosillo, Sonora. Un grupo estuvo configurado por 96 menores con antecedentes de indigencia, de los cuales 94 fueron varones y 2 mujeres, con un promedio de ingreso familiar mensual de $\$ 2,000.00$ (175 dólares) y edades que oscilaron entre 6 y 18 años. El segundo grupo estuvo constituido por 96 menores no indigentes que se encontraban cursando primaria, secundaria y preparatoria, y con similares características demográficas, en cuanto a sexo e ingreso familiar. Para ambos grupos la media de edad fue de 12 años, con escolaridad promedio de quinto grado de primaria. Los menores preferentemente tienen madre y únicamente fueron entrevistadas dos menores del género femenino ya que en la ciudad existe predominio del género masculino en la manifestación del comportamiento indigente (véase Cuadro 1). 
Comportamiento antisocial en menores escolares e indigentes

\section{Cuadro 1}

Medias y desviaciones estándar de variables demográficas

\begin{tabular}{lccc}
\hline Variables & $N$ & Media & $D E$ \\
\hline Edad & 192 & 12.14 & 3.08 \\
Escolaridad & 192 & 5.64 & 3.21 \\
Edad madre & 189 & 33.65 & 15.82 \\
Edad padre & 176 & 34.30 & 19.78 \\
\hline
\end{tabular}

\section{Instrumentos}

Escala de Comportamiento Antisocial. La escala contiene 17 reactivos, seleccionando una opción de siete puntos (Castell, Frías, Corral \& Sotomayor, 2000). Tuvo por objetivo medir la frecuencia con que el menor y sus familiares realizan comportamientos que lesionan a la comunidad en su persona o en sus pertenencias. Estos actos se caracterizan como antisociales (graffiti ${ }^{3}$, lesiones verbales o físicas, hurtos menores o comportamiento adictivo) o como actos delictivos (lesiones físicas que tardan en sanar más de 15 días, homicidio, robo o comercialización de sustancias adictivas), a los que corresponde la aplicación de sentencias penales.

Escalas de Comportamiento Adictivo. Una escala comprende 20 reactivos (Reich \& Herjanic, 1989; Vazsonyi, Pickering, Junger \& Hessing, 2001), seleccionando una opción entre cinco puntos. Tuvo por objetivo identificar la frecuencia con la que el menor y sus familiares consumen drogas o alcohol, especificando cuál es la adicción más usual, ya sea a cigarros, inhalantes, marihuana, cocaína o pastillas estimulantes. La escala de Reich y

3 El comportamiento de graffiti es definido como toda manifestación jeroglífica o pictórica que realizan los menores sobre las paredes de casas o edificios, sin la autorización de los propietarios o las autoridades. 
Herjanic (1989) contó con 11 reactivos, seleccionando una opción de siete puntos, y estuvo orientada a medir la frecuencia con que el menor y sus familiares consumen drogas sin especificar el tipo de sustancia.

Escala del Comportamiento Antisocial del Vecindario. Estuvo integrada por 9 reactivos, seleccionando una opción entre siete puntos (Banda, 2004), que registraron comportamientos antisociales de los vecinos como graffiti, robo y comercialización de drogas, entre otros.

Escala del Comportamiento Adictivo del Vecindario. La escala constó de 8 reactivos, seleccionando una opción entre siete puntos (Banda, 2004). Dicha escala midió la frecuencia del consumo de sustancias adictivas en general realizado por los integrantes del vecindario.

Escala de Violencia Comunitaria. La escala se integró por 15 reactivos de los que se seleccionó una opción entre siete puntos (Banda, 2004). Midió la frecuencia de eventos violentos entre vecinos y entre personas que transitan por el vecindario frente a los que, por la severidad de la agresión, los afectados y las autoridades podrían ejercer demandas legales.

Todo el instrumento se integró por 143 reactivos, los cuales contaron con consistencia interna, habiendo obtenido un alpha de Cronbach superior a .77 .

\section{Procedimiento}

El grupo de menores con antecedentes de indigencia fue entrevistado en las calles en una sola sesión y en albergues públicos 
o privados durante dos sesiones ${ }^{4}$, atendiendo el funcionamiento de los mismos. Los menores no indigentes fueron entrevistados en las escuelas a las que asistían durante varias sesiones, considerando las disposiciones de las mismas. El grupo de menores con antecedentes de indigencia fue identificado mediante un censo realizado en las calles de la ciudad y en albergues. Una vez identificadas las características de este grupo, fue igualado al grupo de menores no indigentes que asistían a la escuela en función de la edad, el sexo y el ingreso económico.

Las entrevistas tuvieron una duración de aproximadamente dos horas para cada menor y fueron llevadas a cabo por psicólogos previamente entrenados en la ejecución de entrevistas y manejo del instrumento.

\section{Análisis de datos}

Se llevaron a cabo análisis de frecuencia para variables categóricas y análisis univariados de las variables continuas. La confiabilidad de las escalas que integraron todas las escalas estudiadas se verificó mediante la obtención de alfas de Cronbach con el paquete estadístico System of Statistical Analysis (SAS) 6.0.

Se sumaron los reactivos (variables) correspondientes al comportamiento antisocial del menor y del padre, los relativos al comportamiento adictivo del menor y de su madre, así como los reactivos relativos al comportamiento antisocial y adictivo del vecindario, y los de la violencia comunitaria fueron convertidos en índices.

4 La sesión se define como el tiempo de aproximadamente cuatro horas en que el entrevistador se mantuvo en las calles o en los albergues, durante el cual podrían haberse realizado una o dos entrevistas. 
Los datos recabados fueron contrastados con el modelo teórico, mediante un modelo de ecuaciones estructurales empleando el paquete estadístico Structural Equations (EQS) 5.7b. El modelo de ecuaciones estructurales permitió cuantificar la relación que mantienen los índices (suma de reactivos) y el factor vecindario con el comportamiento antisocial y adictivo del menor.

El modelamiento de ecuaciones estructurales determinó qué variables integraron los diferentes índices y los índices que formaron el factor vecindario, así como las relaciones causales entre dicho factor, los índices de comportamiento antisocial del padre y el comportamiento adictivo de la madre, y los índices de comportamiento antisocial y adictivo del menor y a su vez la relación causal entre éstos últimos y el factor vecindario.

La bondad de ajuste del modelo para ambos grupos se obtuvo mediante el indicador estadístico Chi cuadrado y mediante los indicadores prácticos: índice de ajuste normado (NFI), índice de ajuste no normado (NNFI) e índice de ajuste comparativo (CFI).

\section{Resultados}

\section{Análisis de frecuencia de medias y confiabilidad de las escalas}

El Cuadro 1 (véase arriba) muestra las medias y desviaciones estándar de las variables demográficas pertenecientes a los menores entrevistados. En total fueron 192 menores de los cuales 96 (50\%) pertenecieron al grupo de menores con antecedentes de indigencia y el resto al grupo de estudiantes (50\%). El grupo estudiado se constituyó por 188 menores de género masculino (98\%) y 4 menores de género femenino (2\%); 153 de estos menores $(80 \%)$ reportaron encontrarse matriculados en escuelas. 


\section{Modelo de ecuaciones estructurales}

La Figura 1 muestra las relaciones que el comportamiento antisocial y adictivo de los menores indigentes establecieron frente a las variables familiares y el factor vecindario. El comportamiento adictivo del menor indigente y el comportamiento antisocial del padre explican el $43 \%$ del comportamiento antisocial de los menores indigentes. El comportamiento antisocial del menor indigente se ve influido directa, positiva y significativamente por el comportamiento adictivo del menor y el comportamiento antisocial del padre e indirectamente por el comportamiento adictivo de la madre y por el factor vecindario. El comportamiento adictivo de la madre y el factor vecindario influyen de forma directa, positiva y significativa en el comportamiento adictivo del menor indigente.

La Figura 2 muestra que el comportamiento antisocial del padre, el factor vecindario y el comportamiento adictivo del menor explican el $46 \%$ del comportamiento antisocial del menor no indigente. El comportamiento antisocial del menor no indigente se encuentra influido positiva, directa y significativamente por el comportamiento antisocial del padre, el factor vecindario y el comportamiento adictivo del menor, el comportamiento adictivo de la madre influye pero indirectamente. Para el grupo de menores no indigentes, el comportamiento adictivo tuvo influencia directa positiva y significativa por parte del factor vecindario y el comportamiento adictivo de la madre. 


\section{Cuadro 2}

Medias y confiabilidad de escalas utilizadas en el estudio para el grupo de menores indigentes

\begin{tabular}{|c|c|c|c|c|}
\hline Escala a medir/ reactivos & $N$ & $\begin{array}{c}\text { Media } \\
\xi\end{array}$ & $D E$ & $\begin{array}{c}\text { Alfa de } \\
\text { Cronbach }\end{array}$ \\
\hline Comportamiento adictivo del menor & & & & .88 \\
\hline Cuánto bebe en cada ocasión & 96 & 0.94 & 1.41 & \\
\hline Frecuencia de consumo de drogas & 96 & 0.93 & 1.76 & \\
\hline Ha fumado cigarros & 96 & 2.08 & 1.73 & \\
\hline Ha fumado marihuana & 96 & 1.00 & 1.73 & \\
\hline Comportamiento antisocial del menor & & & & .92 \\
\hline Salir sin permiso & 94 & 2.53 & 2.32 & \\
\hline Ensuciar calles & 94 & 2.04 & 2.38 & \\
\hline Llegar tarde a casa & 94 & 2.43 & 2.39 & \\
\hline Pelear & 94 & 2.52 & 2.24 & \\
\hline Llevar armas por si pelea & 94 & 1.20 & 1.99 & \\
\hline Robar almacenes & 94 & 1.00 & 1.80 & \\
\hline Pertenecer a pandilla & 94 & 1.17 & 2.11 & \\
\hline Frecuencia con que pelea & 94 & 1.85 & 2.30 & \\
\hline Tomar licor o drogas & 94 & 1.65 & 2.22 & \\
\hline Robar & 94 & 1.56 & 2.21 & \\
\hline Frecuencia con que porta arma & 94 & 2.22 & 2.00 & \\
\hline Comportamiento antisocial del padre & & & & .81 \\
\hline Salir sin avisar & 84 & 1.88 & 2.20 & \\
\hline Llegar tarde & 84 & 1.72 & 2.15 & \\
\hline Pelear con otros & 84 & 1.57 & 2.07 & \\
\hline Frecuencia con que toma licor o drogas & 84 & 1.90 & 2.35 & \\
\hline \multicolumn{5}{|l|}{ Comportamiento adictivo de la madre } \\
\hline Frecuencia con que toma fin semana & 94 & 0.74 & 1.18 & .88 \\
\hline Cuánto bebe en cada ocasión & 94 & 0.76 & 1.29 & \\
\hline Ha fumado cigarros & 94 & 1.15 & 1.73 & \\
\hline Comportamiento antisocial del vecindario & & & & .83 \\
\hline Frecuencia que se pintan las paredes & 96 & 3.25 & 2.31 & \\
\hline Frecuencia se drogan y terminan peleando & 96 & 2.58 & 2.30 & \\
\hline $\begin{array}{l}\text { Frecuencia ingieren alcohol y pelean los } \\
\text { vecinos }\end{array}$ & 96 & 26 & 21 & \\
\hline
\end{tabular}


Comportamiento antisocial en menores escolares e indigentes

Cuadro 2 (continuación)

\begin{tabular}{|c|c|c|c|c|}
\hline Escala a medir/ reactivos & $N$ & $\begin{array}{l}\text { Media } \\
\xi \\
\end{array}$ & $D E$ & $\begin{array}{c}\text { Alfa de } \\
\text { Cronbach }\end{array}$ \\
\hline Frecuencia te invitan a pintar paredes & 96 & 1.04 & 1.82 & \\
\hline $\begin{array}{l}\text { Frecuencia policía organiza redadas en } \\
\text { vecindario }\end{array}$ & 96 & 2.19 & 2.28 & \\
\hline Frecuencia policía busca personas específicas & 96 & 2.17 & 2.28 & \\
\hline Comportamiento adictivo del vecindario & & & & .89 \\
\hline Frecuencia vecinos ingieren bebidas alcohólicas & 96 & 3.13 & 2.28 & \\
\hline Frecuencia vecinos se drogan & 96 & 3.53 & 2.43 & \\
\hline $\begin{array}{l}\text { Frecuencia vecinos ingieren bebidas alcohólicas } \\
\text { entre semana }\end{array}$ & 96 & 3.10 & 2.12 & \\
\hline Frecuencia vecinos ingieren bebidas alcohólicas & & & & \\
\hline los fines de semana & 96 & 3.22 & 2.23 & \\
\hline Frecuencia vecinos se drogan fines de semana & 96 & 3.07 & 2.34 & \\
\hline Frecuencia vecinos te invitan a tomar & 96 & 1.14 & 1.96 & \\
\hline Frecuencia vecinos se drogan durante el día & 96 & 2.41 & 2.46 & \\
\hline Violencia comunitaria & & & & .92 \\
\hline Frecuencia vecinos se pelean & 96 & 2.59 & 2.11 & \\
\hline Frecuencia vecinos se pelean entre ellos & 96 & 2.61 & 2.10 & \\
\hline Frecuencia vecinos se cachetean & 96 & 1.98 & 2.07 & \\
\hline Frecuencia vecinos se empujan & 96 & 2.11 & 2.06 & \\
\hline Frecuencia alguien es golpeado & 96 & 2.30 & 2.18 & \\
\hline Frecuencia alguien es violado & 96 & 1.12 & 1.94 & \\
\hline Frecuencia alguien es asaltado & 96 & 1.71 & 1.99 & \\
\hline Frecuencia aparecen carros desvalijados & 96 & 1.45 & 2.12 & \\
\hline Frecuencia alguien es dañado con arma & 96 & 1.35 & 1.98 & \\
\hline Frecuencia con que se escuchan disparos & 96 & 1.55 & 1.96 & \\
\hline Frecuencia con que hay tiroteos & 96 & 1.22 & 1.83 & \\
\hline Frecuencia con que alguien es herido en riña & 96 & 1.45 & 1.60 & \\
\hline
\end{tabular}




\section{Cuadro 3}

Medias y confiabilidad de escalas utilizadas en el estudio para el grupo de menores no indigentes

\begin{tabular}{|c|c|c|c|c|}
\hline Escala a medir/ reactivos & $N$ & $\begin{array}{l}\text { Media } \\
\xi\end{array}$ & $D E$ & $\begin{array}{l}\text { Alfa de } \\
\text { Cronbach }\end{array}$ \\
\hline Comportamiento adictivo del menor & & & & .18 \\
\hline Menor ha fumado cigarros & 96 & 0.65 & 1.14 & \\
\hline Comportamiento antisocial del menor & & & & .85 \\
\hline Salir sin permiso & 96 & 1.21 & 1.65 & \\
\hline Ensuciar calles & 96 & 1.25 & 1.81 & \\
\hline Llegar tarde a casa & 96 & 1.30 & 1.69 & \\
\hline Pelear & 96 & 1.59 & 1.82 & \\
\hline Comportamiento antisocial del padre & & & & .76 \\
\hline Llegar tarde & 91 & 1.13 & 1.61 & \\
\hline Pelear con otros & 91 & 1.06 & 1.56 & \\
\hline Veces que toma licor o drogas & 91 & 1.27 & 1.93 & \\
\hline Comportamiento adictivo de la madre & & & & .77 \\
\hline Frecuencia madre toma fin semana & 96 & 0.56 & 0.94 & \\
\hline Cuánto bebe mamá en cada ocasión & 96 & 0.51 & 0.80 & \\
\hline Madre ha fumado cigarros & 96 & 0.44 & 1.06 & \\
\hline Comportamiento antisocial del vecindario & & & & .79 \\
\hline Frecuencia que se pintan las paredes & 96 & 2.44 & 2.20 & \\
\hline Frecuencia se drogan y terminan peleando & 96 & 1.54 & 1.73 & \\
\hline Frecuencia ingieren alcohol y pelean & & & & \\
\hline los vecinos & 96 & 1.70 & 1.84 & \\
\hline $\begin{array}{l}\text { Frecuencia policía organiza redadas en } \\
\text { vecindario }\end{array}$ & 96 & 1.29 & 1.86 & \\
\hline Frecuencia policía busca personas & & & & \\
\hline específicas & 96 & 1.07 & 1.40 & \\
\hline Comportamiento adictivo del vecindario & & & & .86 \\
\hline $\begin{array}{l}\text { Frecuencia con que vecinos ingieren bebidas } \\
\text { alcohólicas }\end{array}$ & 96 & 2.68 & 2.18 & \\
\hline Frecuencia con que vecinos se drogan & 96 & 1.81 & 2.04 & \\
\hline $\begin{array}{l}\text { Frecuencia con que vecinos ingieren } \\
\text { bebidas alcohólicas entre semana }\end{array}$ & 96 & 2.14 & 1.94 & \\
\hline
\end{tabular}


Comportamiento antisocial en menores escolares e indigentes

Cuadro 3 (continuación)

\begin{tabular}{lcccc}
\hline Escala a medir/ reactivos & $N$ & $\begin{array}{c}\text { Media } \\
\xi\end{array}$ & DE & $\begin{array}{c}\text { Alfa de } \\
\text { Cronbach }\end{array}$ \\
\hline
\end{tabular}

Frecuencia con que vecinos ingieren bebidas alcohólicas los fines de semana

$96 \quad 2.25 \quad 2.13$

Frecuencia con que vecinos se drogan los

fines de semana

$96 \quad 1.41 \quad 1.85$

Violencia comunitaria

Frecuencia con que vecinos se pelean

Frecuencia vecinos se pelean entre ellos

$\begin{array}{lll}96 & 1.43 & 1.75\end{array}$

$\begin{array}{lll}96 & 1.62 & 1.80\end{array}$

$\begin{array}{llll}\text { Frecuencia con que los vecinos se cachetean } & 96 & 1.02 & 1.60\end{array}$

Frecuencia con que los vecinos se empujan $\quad 96 \quad 1.02 \quad 1.56$

$\begin{array}{llll}\text { Frecuencia con la que alguien es golpeado } & 96 & 1.52 & 1.66\end{array}$

$\begin{array}{llll}\text { Frecuencia con que alguien es asaltado } & 96 & 1.51 & 1.85\end{array}$

Los indicadores de bondad de ajuste de este modelo indican que los datos obtenidos respaldan las propuestas teóricas y empíricas de interacción entre las variables.

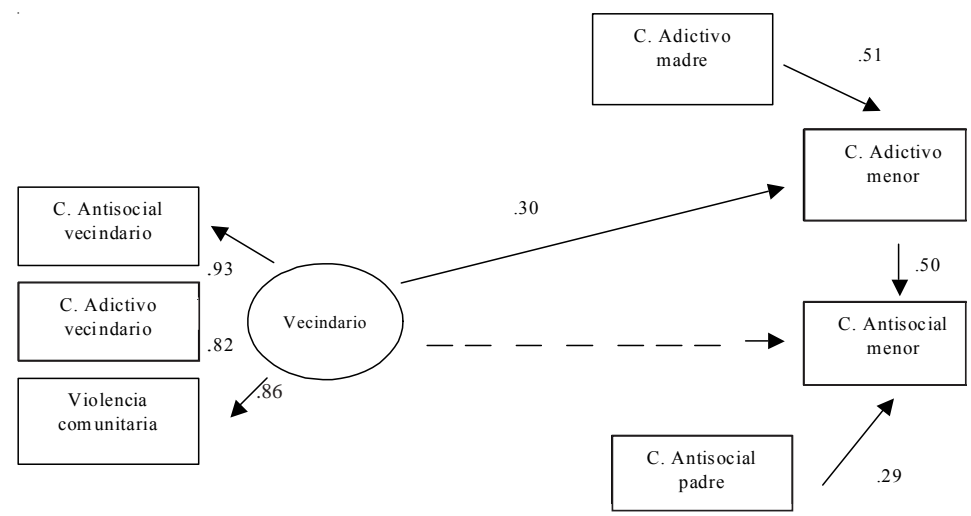

Figura 1. Influencia de la interacción paterna y del vecindario en el comportamiento antisocial y adictivo de menores indigentes.

$$
\begin{aligned}
& \mathrm{c}^{2}=15.56 \mathrm{gl}=10 p=.11 \quad \mathrm{R}^{2}=.43 \\
& I A N=.95 ; \quad I A N N=.96 ; \quad I A C=.98
\end{aligned}
$$

Figura 2. Influencia de la interacción paterna y el vecindario en el 


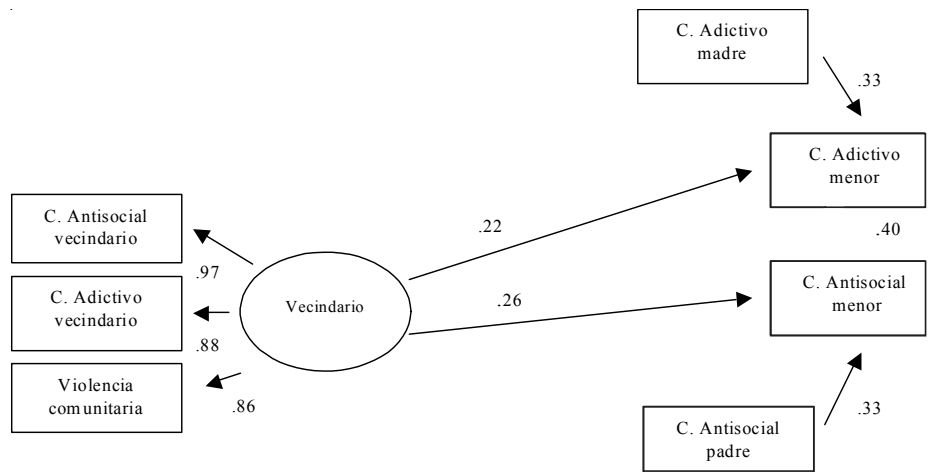

comportamiento antisocial y adictivo de menores no indigentes.

$$
\begin{aligned}
& \mathrm{c}^{2}=50.73 \mathrm{gl}=26 \mathrm{p}=.002 \quad \mathrm{R}^{2}=.46 \\
& I A N=.92 ; \quad I A N N=.94 ; \quad I A C=.96
\end{aligned}
$$

\section{Discusión}

Los datos recabados en la investigación marcan la influencia ejercida por la familia y el vecindario sobre el comportamiento antisocial y adictivo de los menores. Lo que representa que los menores indigentes y no indigentes presentan los mismos, cuando el funcionamiento familiar se encuentra caracterizado por el comportamiento adictivo de la madre y el comportamiento antisocial del padre. Para el grupo de menores no indigentes los vecindarios coadyuvan, para que estos últimos manifiesten comportamientos antisociales y adictivos.

La influencia del comportamiento antisocial del padre y del comportamiento adictivo de la madre conduce a señalar que el comportamiento antisocial de la familia genera influencia en la manifestación del comportamiento antisocial de los menores indigentes, lo cual concuerda con las investigaciones realizadas por NICHD (2003) y Frías (2002). La relación entre padres que muestran comportamiento adictivo y el comportamiento adictivo 
de los menores también se ha localizado en los reportes de Frías (2002), NICHD (2003), Nyamathi et al. (2001) y Stein et al. (2002).

Para el grupo de menores no indigentes se ha encontrado que los vecindarios los afectan directamente, ya que los mismos refieren manifestaciones de comportamiento antisocial y adictivo, lo cual también ha sido puntualizado por Beyers et al. (2001) y Wikström y Loeber (2000), quienes vinculan las condiciones de pobreza de los vecindarios con la antisocialidad de sus integrantes, y por Ceballo et al. (2001) quienes especifican la pauta habitual de la violencia que impera dentro de los vecindarios y la orientación hacia la misma que manifiestan las personas que los habitan.

Las investigaciones realizadas por NICHD (2003), Hussong y Hicks (2003), Garnier y Stein (2002), Schwartz et al. (2002) y Natera et al. (1999) apoyan la influencia del comportamiento de los miembros del vecindario en el comportamiento adictivo de los menores indigentes. No obstante para este mismo grupo el vecindario no ejerce influencia en el comportamiento antisocial.

Para los menores no indigentes el comportamiento del vecindario interviene en el comportamiento antisocial como ya lo han planteado otros autores (Barrera et al., 2002; Galambos et al., 2003; Rosario et al., 2003).

El vecindario y los amigos provenientes del mismo influyen notablemente sobre el comportamiento adictivo de los menores no indigentes, de la misma forma que lo han reportado las investigaciones de Garnier y Stein (2002), Hussong y Hicks (2003), Natera et al. (1999), NICHD (2003) y Schwartz et al. (2000).

Al igual que las investigaciones de Bulos y Ávalos (1993), Frías (2002), Frías, Corral et al. (2000) y Natera et al. (1999) esta 
investigación encontró que el comportamiento adictivo de los menores constituye una variable mediadora. Por consiguiente, el comportamiento adictivo suele promover el comportamiento antisocial para ambos grupos de menores indigentes y no indigentes.

Finalmente, cabe señalar que el desarrollo del comportamiento adictivo y antisocial de los menores no puede ser enteramente atribuible a la influencia ejercida por los padres, ya que es importante considerar que el resto del entorno, particularmente los integrantes del vecindario sean miembros de su grupo de amistades o no, también son susceptibles de afectar a los menores y a sus familias, de la misma forma que lo han planteado Pinderhughes et al. (2001) y Tolan et al. (2003).

\section{Referencias}

Banda, A. L. (2004). Indigencia infantil y conducta antisocial: un modelo ecológico. En M. Frías \& V. Corral (Eds.), Delincuencia juvenil (pp. 101-126). México: CONACYT/Universidad de Sonora.

Barrera, M., Jr., Prelow, H., Dumka, L., Gonzales, N., Knight, G., Michaels, M. et al. (2002). Pathways from family economic conditions to adolescents' distress: Supportive parenting, stressors outside the family, and deviant peers. Journal of Community Psychology, 30, 2, 135-152.

Beyers, J., Loeber, R., Wikstrom, P. \& Southamer-Loeber, M. (2001). What predicts adolescent violence in better-off neighborhoods? [versión electrónica], Journal of Abnormal Child Psychology. Recuperado el 16 de marzo de 2003, de http://www.findarticles. com/cf_o/m0902/5_29/80056062/p1/article.jhtml?tem= BRONFENBRENNER

Bronfenbrenner, U. (1987). La ecología del desarrollo humano. España: Paidós. 
Bulos, C. \& Ávalos, C. (1993). La percepción comunitaria e institucional de la farmacodependencia en el DIF. En Consejo Nacional contra las Adicciones (Ed.), Las adicciones: hacia un enfoque multidisciplinario (pp. 70-76). México: Secretaría de Salud.

Castell, I., Frías, M., Corral, V. \& Sotomayor, M. (2000). Apoyo familiar, castigo corporal y sus repercusiones conductuales en niños. Revista Mexicana de Psicología, 17, 1, 37-45.

Ceballo, R., Dahl, T. A., Aretakis, M. T. \& Ramirez, C. (2001). Innercity children's exposure to community violence: How much do parents know? Journal of Marriage and Family, 63, 4, 927-940.

Early Child Care Research Network Rockville Maryland. (2003). Do children's attention processes mediate the link between family predictors and school readiness? Developmental Psychology, 39, 3, 581-593.

Fergusson, D. \& Horwood, J. (1998). Exposure to interparental violence in childhood and psychosocial adjustment in young adulthood. Child Abuse \& Neglect, 22, 5, 339-357.

Frías, M. (2002). Long-term effects of child punishment on Mexican women: A structural model. Child Abuse \& Neglect, 26, 371-386.

Frías, M. (2004). Evaluación empírica de los supuestos de la ley en materia de justicia juvenil. En M. Frias \& V. Corral (Eds.), Delincuencia juvenil (pp. 61-83). México: CONACYT-UNISON.

Frías, M., Corral, V., Moreno, C. \& Rodríguez. I. (2000). El maltrato infantil como un factor de riesgo para la salud, la conducta prosocial y la ejecución escolar en niñas y niños. Investigaciones Educativas en Sonora, 2, 32-65.

Frías, M., Figueredo, A. J., Corral, V., Peña, E. \& Quiroz, A. (2002). Eficacias sociales, influencias paternas y antisocialidad juvenil: un modelo evolucionista. Enseñanza e Investigación en Psicología, 7, 1, 139-159.

Frías, M., López, A. E. \& Díaz, S. G. (2003). Predictores de la conducta antisocial juvenil: un modelo ecológico. Estudios de Psicología (Natal). Recuperado 23 de enero de 2004, de http://wwwscielo.br/ 
scielo.php2script=sci_arttext8pid=s1413-294x2003000100003\& Ing=en\&nrm=iso

Frías, M., Ramírez, J., Soto, R., Castell, I. \& Corral, V. (2000). Repercusiones del maltrato infantil: un estudio con niños en alto riesgo de maltrato. La Psicología Social en México, VIII, 479-485.

Frías, M., Sotomayor, M., Varela, C., Zaragoza, F., Banda, A. L. \& García, A. (2000). Predictores de la delincuencia juvenil. La Psicología Social en México, VIII, 486-492.

Frías, M., Sotomayor, M., Corral, V. \& Castell, I. (2004). Parental styles and harsh parenting in a sample of Mexican women: A structural model. Revista Interamericana de Psicología, 38, 1, 61-72.

Galambos, N. L., Barker, E. T. \& Almeida, D. M. (2003). Parents do matter: Trajectories of change in externalizing and internalizing problems in early adolescence. Child Development, 74, 2, 578-594.

Garbarino, J. (2001). An ecological perspective on the effects of violence on children. Journal of Community Psychology, 29, 3, 361-378.

Garnier, H. E. \& Stein, J. A. (2002). An 18-year model of family and peer effects on adolescent drug use and delinquency. Journal of Youth and Adolescence, 31, 1, 45-56.

Hussong, A. M. \& Hicks, R. E. (2003). Affect and peer context interactively impact adolescent substance use. Journal of Abnormal Child Psychology. Recuperado 29 de junio de 2004, de http://www.findarticles.com/mi_m0902/is_4_31/ai_104634485

Instituto Nacional de Estadística, Geografía e Informática (INEGI). (2002). Estadísticas Judiciales en Materia Penal No.10. En INEGI (Ed.), Presuntos delincuentes registrados en los juzgados de la instancia del fuero común por principales tipos de delito según entidad federativa de ocurrencia, grupo de edad y sexo 2001 (pp. 9-25). México: INEGI.

Instituto Nacional de Estadística, Geografía e Informática (INEGI). (2002). Anuario Estadístico de los Estados Unidos Mexicanos. 
En INEGI (Ed.), Casos puestos a disposición del Consejo Tutelar 2001 (pp. 197). México: INEGI.

Mcevoy, A. \& Welker, R. (2000). Antisocial behavior, academic failure and school climate: A critical review. Journal of Emotional and Behavioral Disorders. Recuperado el 10 de diciembre de 2003, de http://www.findarticles.com/cf_0/ m0fcb/3_8/66107262/print.jhtml

Natera, G., Mora, J. \& Tiburcio, M. (1999). Barreras en la búsqueda de apoyo social para las familias con problemas de adicciones. Salud Mental, 22, Esp., 114-120.

National Institute on Drug Abuse [NIDA] (2006). Criteria for substance, dependence and diagnosis. Recuperado el 31 de enero de 2006 de http://www.nida.nih.gob/Drugpages/ DSR.html

Nyamathi, A., Longshore, D., Keenan, C., Lesser, J. \& Leake, B. D. (2001). Childhood predictors of daily substance use among homeless women of different ethnicities. American Behavioral Scientist, 45, 1, 35-50.

Reich, W. \& Herjanic, B. (1989). The diagnostic interview for children and adolescents. Manuscrito no publicado, Universidad de Washington en St. Louis, MO, EE. UU.

Rosario, M., Salzinger, S., Feldman, R. S. \& Nq-Mak, D. S. (2003). Community violence exposure and delinquent behaviors among youth: The moderating role of coping. Journal of Community Psychology, 31, 5, 489-512.

Schwartz, D., Dodge, K. A., Pettit, G. S. \& Bates, J. E. (2000). Friendship as a moderating factor in the pathway between early harsh home environment and later victimization in the peer group. Developmental Psychology, 36, 5, 646-662.

Stein, J. A., Burden Leslie, M. \& Nyamathi, A. (2002). Relative contributions of parent substance use and childhood maltreatment to chronic homelessness, depression, and substance abuse problems among homeless women: Mediating roles of selfesteem and abuse in adulthood. Child Abuse \& Neglect, 26, 10, 1011-1027. 
Tolan, P. H., Gorman-Smith, D. \& Henry, D. B. (2003). The developmental ecology of urban males' youth violence. Developmental Psychology, 39, 2, 274-291.

Tremblay, R. E., Pihl, R. O., Vitaro, F. \& Dobkin, P. L. (1999). Patrons de comportements en maternelle et émergence précoce de comportements antisociaux chez les garçons. Bulletin de Psychologie, 52, 3, 373-382.

Tyler, K. A. \& Cause, A. M. (2002). Perpetrators of early physical and sexual abuse among homeless and runaway adolescent. Child Abuse \& Neglect, 26, 12, 1261-1274.

Vazsonyi, A., Pickering, L., Junger, M. \& Hessing, D. (2001). An empirical test of a general theory of crime: A four-nation comparative study of self-control and the prediction of deviance. Journal of Research in Crime and Delinquency, 38, 2, 91-131.

Velasco, R. (2003). El modelo integral. En R. Velasco (Ed.), Adicciones (pp. 13-29). México: Trillas.

Wikström, P. \& Loeber, R. (2000). Do disadvantage neighborhoods cause well-adjusted children to become adolescent delinquents? A study of male juvenile serious offending, individual risk and protective factors and neighborhood context. Criminology, 38, 4, 1109-1141. 